\title{
Searching for secondary findings: considering actionability and preserving the right not to know
}

\author{
Bertrand Isidor ${ }^{1}$ - Sophie Julia ${ }^{2}$ Pascale Saugier-Veber ${ }^{3,4} \cdot$ Paul-Loup Weil-Dubuc $^{5}$ - Stéphane Bézieau ${ }^{1}{ }^{1}$. \\ Eric Bieth ${ }^{2} \cdot$ Jean-Paul Bonnefont ${ }^{6} \cdot$ Arnold Munnich $^{6} \cdot$ Franck Bourdeaut $^{7} \cdot$ Catherine Bourgain $^{8}$. \\ Nicolas Chassaing ${ }^{2}$ - Nadège Corradini ${ }^{9} \cdot$ Damien Haye $^{10} \cdot$ Julie Plaisancie $^{2} \cdot$ Delphine Dupin-Deguine $^{2,11}$. \\ Patrick Calvas $\mathbb{D}^{2} \cdot$ Cyril Mignot $^{10}$ - Benjamin Cogné ${ }^{\prime}$. Sylvie Manouvrier ${ }^{12,13}$ - Laurent Pasquier $\mathbb{D}^{14}$. \\ Delphine Héron ${ }^{10} \cdot$ Kym M Boycott ${ }^{15}$ - Mauro Turrini $\mathbb{1}^{16}$. Danya F. Vears $\mathbb{D}^{17,18,19,20}$. Mathilde Nizon ${ }^{1}$. \\ Marie Vincent $\mathbb{1}^{1}$
}

Received: 8 February 2019 / Revised: 4 May 2019 / Accepted: 21 May 2019 / Published online: 11 June 2019

(c) The Author(s), under exclusive licence to European Society of Human Genetics 2019

Secondary findings (SF) differ from incidental findings as they are actively sought and systematically evaluated using a list of genes selected based on guidelines developed by professional societies in various jurisdictions. Despite some authors stating that a "consensus regarding the return of secondary genomic findings in the clinical setting has been reached" [1], we believe that further consideration of the issue is required. In particular, given the absence of scientific evidence of pathogenicity of these allegedly causative variants in unaffected individuals, the question remains as to how beneficial the knowledge of these variants is to the patient, and his/her relatives.

Although guidelines issues by European professional bodies do not specifically address SF, their call for a targeted approach to analysing genomic data in relation to clinical question implies that they are not supportive of actively searching for disease-causing variants extraneous to the phenotype of the patient $[2,3]$. In contrast, while the American College of Medical Genetics and Genomics (ACMG) Working Group "acknowledge that there was insufficient evidence about benefits, risks and costs of disclosing incidental findings to make evidence-based recommendations", their final recommendation was to report SF on their "list" [4]. This is based on the perceived "actionable" nature of such information and therefore the value of sharing this information. Yet, recent studies have revealed that variants formerly recognised as pathogenic are

Bertrand Isidor

bertrand.isidor@chu-nantes.fr

Marie Vincent

marie.vincent@chu-nantes.fr

Extended author information available on the last page of the article present in disease-free individuals [5]. Identifying a predisposing variant in an affected individual or in a family where several members are affected by a serious condition, makes medical and scientific sense. But evaluating the risk associated with such a variant when it is identified outside of any pathological context is currently very challenging. This could lead to an increased number of unnecessary follow-up investigations, needless anxiety for patients, and poor medical decisions. To date, no scientific study has systematically evaluated the benefit of preventive measures in response to this kind of information in this context.

Actionability has become the criterion used to determine whether or not to report SF. Unfortunately, this term has been used to describe various concepts, such as "preventable or treatable conditions" [6], "potential to change immediate medical care" [7], and "a finding that may impact patient management to improve outcome" [8], among others. One definition that might prove useful is that of Richer and Laberge: "a pathogenic variant for which preventive and/or treatment measures are available to significantly improve health outcomes associated with the condition" [9]. However, as these authors acknowledge, the definition still requires a determination of what constitutes a significant impact on health outcomes. Furthermore, actionability carries with a positive connotation or value, as it is also the term used in tumour genomics to qualify the somatic variants considered as biomarkers for targeted therapies. Using this term might bias or skew the discussion between clinicians and patients or their families. To reach a consensus as a community we need to determine actionability with more objective measures. While some authors have proposed a semiquantitative metric to assess actionability [10], reference data obtained from asymptomatic or symptomatic (for another condition) individuals who carry 
these variants is lacking. Instead, one could analyse the ACMG list of 59 "actionable" genes in the general population and compare medical, psychological, and social outcomes of those who did and did not receive medical care in response to variants identified.

In France, a strict legal framework for presymptomatic testing has been developed, which includes a period of reflection and involvement of a multidisciplinary team, including a psychologist. These measures, which allow for patient autonomy, are not questioned, either by geneticists or by individuals receiving testing [11]. Yet, the introduction of an active search for SF as part of the protocol for diagnostic sequencing would mean any member of the population undergoing testing for a particular indication could receive predictive health-related information. This is regardless of whether they have a family history of the condition or any previous concerns about the diseases being screened. It seems illogical that the highly refined processes associated with existing predictive testing protocols would be completely eclipsed by the availability of SF when sequencing is performed for an unrelated indication.

In addition, asymptomatic individuals, or individuals symptomatic for another condition, found to carry predisposing variants may not benefit from the same medical care as affected individuals carrying the same predisposing variants. In other words, "actionability" may differ when considering the potential for medical care offered either to symptomatic individuals with a definitive diagnosis, or to asymptomatic individuals identified and tested based a positive family history. Furthermore, the boundaries of "non-actionability" are not very clearly defined. Even in the cases when there is no effective therapy, non-therapeutic measures can be taken which can deeply modify the way people anticipate their future lives.

High-throughput sequencing techniques are now becoming more widely available and the new French Genomic Medicine Plan will provide tens of thousands of patients every year with access to this testing [12]. Before carrying out genomic analysis, patients will have been counselled by a health professional (not necessarily with genetic training) about the testing, including the potential for SF to be identified during the analysis. Having given voluntary and "informed" consent, all results, both those related to the initial indication for testing and any SF, will, therefore, be released to the patient. Yet every physician who gains consent from their patients is well aware of its limitations: crippling time constraints, difficulties in comprehension due a lack of knowledge or language barriers, etc. Based on our current knowledge, it is difficult to predict, let alone control the possible future impact of SF and one cannot truly consider the medical, familial, social, professional and psychological consequences of such a result. As a community, we are well aware of the problems arising from consultations involving presymptomatic diagnosis when these concern one well-identified disease already known to exist in the family. How can we, possibly inform patients of the potential detection of numerous diseases identifiable via this type of genomic analysis? Like others [13], we question whether patients will be able to comprehend the goals of the test and the implications or impact of the results, and therefore make an informed decision about whether or not they want to receive them. We observe this in our practice and we try to manage it whilst attempting to respect the autonomy of patients and their families. However, in our opinion, the significant gaps in knowledge about the real impact of SF by the medical genetics community will, by extension, result in an inability to truly enable a patient to provide informed consent for SF.

We also need to consider how free patients are to exercise their "right not to know" SF when they are told that this kind of analysis is an option. Studies exploring patients' and publics' views about receiving secondary and unsolicited findings show that, in hypothetical situations, high proportions of people want to receive all kinds of findings from genomic sequencing, in some cases regardless of whether or not they are actionable [14]. However, there is little data to date exploring whether these preferences are expressed and maintained by actual patients after testing takes place.

In addition, in France, there is legislation relating to the disclosure of genetic information to at-risk relatives [15]. This legislation does stress that if the genetic condition identified is serious and either treatable or preventable (including genetic counselling), patients should either disclose these to their at-risk relatives, or give permission for a healthcare professional to do so on their behalf. This means that identification of SF carries with it the legal obligation to inform other at-risk family members. This pressure for the patient, or their healthcare professional, to disclose this information to at-risk relatives may place an additional burden on the family and should be considered as part of the informed consent process.

The direct consequence of deciding to actively search for SF would be to make them accessible to any individual desirous of knowing their status from a list of "actionable" genes. This decision would also modify the illness definition from clinical to molecular, which might, in an open future, transform everyone into a "patient-in-waiting" [16]. We must consider the applicability of US-centric guidelines to the diversity of European cultures and legal frameworks before integrating this "opportunistic screening" into routine practice.

\section{Compliance with ethical standards}

Conflict of interest The authors declare that they have no conflict of interest. 
Publisher's note: Springer Nature remains neutral with regard to jurisdictional claims in published maps and institutional affiliations.

\section{References}

1. Sapp JC, Johnston JJ, Driscoll K, Heidlebaugh AR, Miren Sagardia A, Dogbe DN.NISC Comparative Sequencing Program et al. Evaluation of recipients of positive and negative secondary findings evaluations in a hybrid CLIA-research sequencing pilot. Am J Hum Genet. 2018;103:358-66.

2. Van ElCG, Cornel MC, Borry P, Hastings RJ, Fellmann F, Hodgson SV.ESHG Public and Professional Policy Committee et al. Whole-genome sequencing in health care. Recommendations of the European Society of Human Genetics. Eur J Hum Genet. 2013;21(Suppl 1):S1-5.

3. Matthijs G, Souche E, Alders M, Corveleyn A, Eck S, Feenstra I. EuroGentest; European Society of Human Genetics et al. Guidelines for diagnostic next-generation sequencing. Eur J Hum Genet. 2016;24:2-5.

4. Kalia SS, Adelman K, Bale SJ, Chung WK, Eng C, Evans JP, et al. Recommendations for reporting of secondary findings in clinical exome and genome sequencing, 2016 update (ACMG SF v2.0): a policy statement of the American College of Medical Genetics and Genomics. Genet Med. 2017;2:249-55.

5. Chen R, Shi L, Hakenberg J, Naughton B, Sklar P, Zhang J, et al. "Analysis of 589,306 genomes identifies individuals resilient to severe Mendelian childhood diseases.". Nat Biotechnol. 2016;34:531-8

6. Zawati MH, Parry D, Thorogood A, Nguyen MT, Boycott KM, Rosenblatt D, et al. Reporting results from whole-genome and whole-exome sequencing in clinical practice: a proposal for Canada? J Med Genet. 2014;51:68-70.

7. Fullerton SM, Wolf WA, Brothers KB, Clayton EW, Crawford DC, Denny JC, et al. Return of individual research results from genome-wide association studies: experience of the Electronic
Medical Records and Genomics (eMERGE) Network. Genet Med. 2012;14:424-31.

8. Boycott K, Hartley T, Adam S, Bernier F, Chong K, Fernandez $\mathrm{BA}$, et al. The clinical application of genome-wide sequencing for monogenic diseases in Canada: Position Statement of the Canadian College of Medical Geneticists. J Med Genet. 2015; 52:431-7.

9. Richer J, Laberge AM. Secondary findings from next-generation sequencing: what does actionable in childhood really mean? Genet Med. 2018. https://doi.org/10.1038/s41436-018-0034-4.

10. Berg JS, Foreman AK, O'Daniel JM, Booker JK, Boshe L, Carey $\mathrm{T}$, et al. A semiquantitative metric for evaluating clinical actionability of incidental or secondary findings from genome-scale sequencing. Genet Med. 2016;18:467-75.

11. Godino L, Turchetti D, Jackson L, Hennessy C, Skirton H. "Impact of presymptomatic genetic testing on young adults: a systematic review.”. Eur J Hum Genet. 2016;24:496-503.

12. Pasquier L, Isidor B, Rial-Sebbag E, Odent S, Minguet G, Moutel G. "Population genetic screening: current issues in a European country." Eur J Hum Genet. 2019. https://doi.org/10.1038/s41431019-0425-2.

13. Viberg J, Segerdahl P, Langenskiöld S, Hansson MG. Freedom of Choice About Incidental Findings Can Frustrate Participants' True Preferences. Bioethics . 2016;30:203-9.

14. Bennette CS, Trinidad SB, Fullerton SM, Patrick D, Amendola L, Burke W, et al. Return of incidental findings in genomic medicine: measuring what patients value-development of an instrument to measure preferences for information from next-generation testing (IMPRINT). Genet Med. 2013;15:873-81.

15. Décret $\mathrm{N}^{\circ} 2013-527$ du 20 juin 2013 relatif aux "conditions de mise en œuvre de l'information de la parentèle dans le cadre d'un examen des caractéristiques génétiques à finalité médicale". French public health code.

16. Timmermans S, Buchbinder M. Patients-in-waiting: Living between sickness and health in the genomics era. J Health Soc Behav. 2010;51:408-23.

\section{Affiliations}

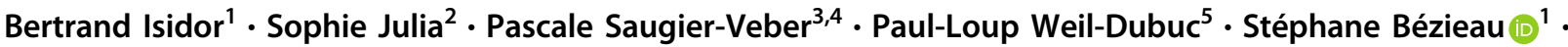 Eric Bieth ${ }^{2} \cdot$ Jean-Paul Bonnefont ${ }^{6} \cdot$ Arnold Munnich $^{6} \cdot$ Franck Bourdeaut $^{7} \cdot$ Catherine Bourgain $^{8}$. Nicolas Chassaing ${ }^{2} \cdot$ Nadège Corradini $^{9} \cdot$ Damien Haye $^{10} \cdot$ Julie Plaisancie $^{2} \cdot$ Delphine Dupin-Deguine $^{2,11}$. Patrick Calvas $\mathbb{D}^{2} \cdot$ Cyril Mignot $^{10}$ - Benjamin Cogné ${ }^{2}$ Sylvie Manouvrier ${ }^{12,13}$ - Laurent Pasquier $\mathbb{D}^{14}$. Delphine Héron ${ }^{10} \cdot$ Kym M Boycott ${ }^{15} \cdot$ Mauro Turrini $\mathbb{i}^{16} \cdot$ Danya F. Vears $\mathbb{(}^{17,18,19,20} \cdot$ Mathilde Nizon ${ }^{1}$. Marie Vincent $\mathbb{( D}^{1}$}

1 Service de génétique médicale, CHU Nantes, 9 quai Moncousu, 44093 Nantes, France

2 Service de génétique médicale, Hôpital Purpan, Centre Hospitalier Universitaire, 31059 Toulouse, France

3 Normandie Univ, UNIROUEN, Inserm U1245, Normandy Centre for Genomic and Personalized Medicine, 76000 Rouen, France

4 Department of Genetics, Rouen University Hospital, Normandy Centre for Genomic and Personalized Medicine, 76000 Rouen, France

5 Espace éthique Ile-de-France, Laboratoire d'excellence Distalz, Université Paris-Sud, Paris-Saclay, France
6 Service de génétique médicale, Hôpital Necker-Enfants Malades, AP-HP, Paris, France

7 Institut Curie, Paris, France

8 Cermes3 (Centre de recherche médecine, sciences, santé, santé mentale, société), Inserm U988, site CNRS, 7 rue Guy Môquet, 94801 Villejuif, France

9 Institut d'hémato-oncologie pédiatrique, Centre Léon Bérard, Lyon, France

10 APHP, Département de Génétique, Groupe Hospitalier Pitié Salpêtrière, Paris, France 
11 Service d'otoneurologie et ORL pédiatrique, Hôpital Purpan, Centre Hospitalier Universitaire, 31059 Toulouse, France

12 Clinique de génétique, CHU de Lille, 59000 Lille, France

13 EA7364 Faculté de Médecine Université de Lille, 59000 Lille, France

14 CHU Rennes, Service de Génétique Clinique, 16 Boulevard de Bulgarie, 35203 Rennes, France

15 Children's Hospital of Eastern Ontario Research Institute, University of Ottawa, Ontario, Canada

16 DCS- Droit et Changement Social Université de Nantes, Nantes, France
17 Department of Public Health and Primary Care, Center for Biomedical Ethics and Law, KU Leuven, Belgium

18 Leuven Institute for Human Genetics and Society, Leuven, Belgium

19 Melbourne Law School, University of Melbourne, Carlton, Australia

20 Murdoch Children's Research Institute, The Royal Children's Hospital, Parkville, Australia 